\title{
EFEITO DO TAMANHO DE FRUTO E DO MÉTODO DE EXTRAÇÂO NA QUALIDADE FISIOLÓGICA DE SEMENTES DE PORONGO
}

\author{
EFFECT OF FRUIT SIZE AND EXTRACTION METHOD ON THE PHYSIOLOGICAL \\ QUALITY OF BOTTLE GOURD SEEDS
}

\author{
Dilson António Bisognin ${ }^{1}$ Nilson Lemos de Menezes ${ }^{2}$ Rogério António Bellé \\ Adriana Maia Albini ${ }^{3}$
}

\section{RESUMO}

Frutos de porongo - Lagenaria siceraria (Mol.) Standi - colhidos de uma lavoura localizada no município de Restinga Seca, RS, foram utilizados para estudar a qualidade fisiológica das sementes. O experimento foi conduzido no Laboratório de Análise de Sementes de Produção, da Universidade Federal de Santa Maria, com sementes extraídas de 10 frutos grandes, médios e pequenos. Os frutos foram caracterizados pelo comprimento, diâmentro, espessura de casco e pelo número de sementes; e as sementes foram caracterizadas pelo comprimento, largura, espessura e peso de 1000 sementes. Frutos médios armazenados por 60 dias foram utilizados para comparar métodos de extração de sementes. Os métodos estudados foram: fermentação em água por 24, 48, 72, 96 e 120h e imersão em solução de ácido clorídrico $(\mathrm{HCl})$ por $30 \mathrm{~min}$. nas concentrações de 10, 20 e $30 \%$. A testemunha consistiu na retirada manual da placenta aderida às sementes. A qualidade fisiológica das sementes foi avaliada através do teste de germinação, primeira contagem e tempo médio de germinação. Os resultados indicam que o tamanho de fruto de porongo pode ser caracterizado pelo seu comprimento ou diâmetro. Sementes mais leves, devido a sua menor largura e espessura, foram extraídas de frutos de menor tamanho. $\mathrm{O} \mathrm{HCl}$ não foi eficiente para a extração de sementes de porongo, pois não facilitou a limpeza e afetou, principalmente nas concentações de 20 e 30\%, a germinação $e$ o vigor das sementes. Concluiu-se que quanto maior o porongo maior é o número e peso de suas sementes, sem diferenca na capacidade de produzir plântulas normais; a limpeza de sementes de porongo através da imersão em solução contendo $\mathrm{HCl}$ reduz o vigor; o melhor tempo de fermentação para as sementes de porongo em água à $25^{\circ} \mathrm{C} e ́$ as $72 \mathrm{~h}$. no entanto o período de 48 a $96 \mathrm{~h}$ melhora a qualidade fisiológica, atém de facilitar a limpeza; e as sementes de porongo, em condições favoráveis de temperatura, umidade e aeração. germinam após terem atingido 43,4\% de umidade.

Palavras-chave: germinação, vigor, Lagenaria siceraria.

\section{SUMMARY}

Bottie gourd - Lagenaria siceraria (Mol.) Standl - the fruits harvested from a farm localed in Restinga Seca county, were used to evaluate seeds in the Seed Laboratory of the Federal University of Santa Maria. The effect of fruit size on seed physiological quality was studied using seeds from ten large, medium and small bottle gourd fruits. Fruits were characterized by the length, the diameter. the shell thickness, and by the number of seeds. The seeds were characterized by the length, the width, the thickness, and the weight of 1000 seeds. Water fermentation during 24, 48, 72, 96, and 120 hours and dipping in $\mathrm{HCl}$ solution by 10, 20, and 30\% during 30 minutes were used to compare methods of extracting seeds from medium bottle gourd fruits stored for 60 days. The manual extraction of seeds was considered as the control treatment. The physiological quality of the seeds was evaluated by the germination and the first counting test and averaging time of germination parameters. Results indicated that the size of botlle gourd fruit can be characterized either by the length or the width.

\footnotetext{
${ }^{1}$ Engenheiro Agrônomo, Mestre, Professor do Departamento de Fitotecnia, Universidade Federal de Santa Maria (UFSM), 97119-900,

Santa Maria, RS. (Auotr para correspondência).

${ }^{2}$ Engenheiro Agrônomo, Doutor, professores do Departamento de Fitotecnia, UFSM

${ }^{3}$ Acadêmica do Curso de Agronomia, UFSM.
} 
Lighter seeds with smaller width and thickness were extracted from smaller fruits. The treatment with $\mathrm{HCl}$ solution specially on 20 and $30 \%$ concentration was not efficient for the extracting bottle gourd seeds because the cleaning process was more difficult and qffècted negatively seed germination and the vigor. The results show that as the size of the fruits increase the number and weight of seeds also increase without changing the ability to produce normal seedlings. The cleaning process of bottle gourd seeds in $\mathrm{HCl}$ solution reduces their vigor. The best period of fermentation of seeds was observed in water at $25^{\circ} \mathrm{C}$ for 72 hours, however, the period from 48 to 96 hours improved their physiological quality and also facilitated the cleaning process. The bottle gourd seeds germinate in environment with $43.4 \%$ of humidity and resonable air temperature, humidity and aeration.

Key words: germination, vigor, Lagenaria siceraria.

\section{INTRODUÇÃO}

O porongo, Lagenaria siceraria (Mol.) Standi, planta da família das cucurbitáceas, é de grande importância para os pequenos agricultores do Rio Grande do Sul, principalmente da região de Santa Maria, onde se concentra o maior número de indústrias de beneficiamento do fruto (BISOGNIN et al., 1995). Esta espécie apresenta crescimento indeterminado, o que acarreta uma grande desuniformidade nos estádios de maturidade dos frutos, podendo se refletir no tamanho dos mesmos e na qualidade fisiológica das sementes. A produção de frutos de menor tamanho pode ser devido a fatores genéticos e/ou ambientais. BISOGNIN \& MARCHEZAN (1988) observaram grande variabilidade genética nas populações utilizadas de porongo, o que resulta na produção de frutos de diversos formatos e tamanhos, sendo muitos deles rejeitados pela indústria de cuia. A senescência natural das plantas, que interrompe o crescimento do fruto, também pode resultar na produção de frutos de menor tamanho e de menor espessura de casco.

Em outras espécies de cucurbitáceas, a maturidade dos frutos está altamente relacionada com a germinação e o vigor das sementes, quando colhidas imaturas, apresentam baixa germinação e vigor (ARAÚJO et al., 1982; ALVARENGA et al, 1984).Outro aspecto a ser considerado, é que nos primeiros 30 dias após a colheita as sementes de cucurbitáceas podem apresentar dormência ou germinação muito lenta (CASALI et al., 1982).

As sementes de porongo estão localizadas na parte apical do fruto, não utilizada pela indústria de cuia, facilitando a extração das sementes. Desta forma, o corte do fruto para a obtenção da cuia expõe as sementes que estão aderidas a placenta. As sementes, quando são secas juntamente com a placenta, ficam aderidas e formam aglomerados que dificultam a limpeza e processamento podendo, ainda, servir de substrato para o crescimento de microrganismos (NASCIMENTO et al., 1994).

A remoção da placenta aderida à semente pode ser feita através da fermentação natural e por processos mecânicos ou químicos (NASCIMENTO et al., 1994). A escolha do método de extração das sementes, bem como da sequência de operações, é função das características do fruto; da maneira como as sementes se encontram associadas as demais partes do fruto; da presença de mucilagem; da presença de patógenos transmissíveis pelas sementes; do volume de frutos; da tolerância da semente à desidratação; e da finalidade ou destino da polpa do fruto (SILVA, 1983).

O processo de fermentação natural vem sendo empregado a muito tempo com a finalidade de degradar a placenta que envolve as sementes, facilitando a lavagem, sendo que a temperatura e o tempo de fermentação pode influenciar o vigor e a germinação das sementes de diferentes espécies. Sementes de pepino fermentadas por 4 a 6 dias mantiveram sua germinação acima de $95 \%$, em teste realizado 90 dias após a extração (COUTO et al., 1969). Para o caso do tomate, as sementes devem ser fermentadas por 3 a 5 dias, com temperatura na faixa de $21-27^{\circ} \mathrm{C}$ (SILVA, 1983).

O tratamento químico com ácido clorídrico (HC1) tem sido bastante usado para separar sementes da placenta ou da sua mucilagem. A rapidez do processo aliada a eficiência da degradação da mucilagem são as características mais importantes do método. COUTO et al. (1969) utilizaram o ácido clorídrico comercial (38\%), por 30 minutos, na proporção de 7,51/t de frutos triturado e peneirado, para a extração de sementes de pepino e obtiveram uma extração mais rápida, além de que as sementes se apresentaram mais claras em relação aos demais tratamentos utilizados.

O objetivo deste trabalho foi estudar a relação entre tamanho de fruto e a qualidade fisiológica de sementes de porongo, bem como estabelecer um método eficaz e adaptado para a extração das sementes.

\section{MATERIAIS E MÉTODOS}

Os frutos que formaram os lotes de sementes utilizadas para execução deste trabalho provieram de uma lavoura localizada no município de Restinga Seca, RS. A semeadura foi realizada no dia 18/09/94, na densidade de 13.300 plantas/ha e a adubação foi de $150 \mathrm{~kg} /$ ha de NPK da fórmula $5-20$ 20. A colheita dos frutos foi realizada dia 15/3/95, no momento da senescência natural das plantas. 
Experimento I - Utilizaram-se três lotes de sementes oriundas de 10 frutos de cada tamanho, separados visualmente em pequeno, médio e grande. Em cada fruto foram realizadas as seguintes medidas: comprimento, diâmetro da parte posterior (onde localizam-se as sementes) e espessura do casco (três determinações equidistantes no local de corte do fruto para obter a cuia). As sementes de frutos do mesmo lote foram retiradas, contadas e misturadas para serem avaliadas. As sementes foram caracterizadas pelo número médio por fruto, peso de 1000 sementes, comprimento, largura e espessura (determinados em 20 sementes). A umidade das sementes foi determinada pelo método de estufa com temperatura de $105^{\circ} \mathrm{C} \pm 3^{\circ} \mathrm{C}$, conforme indicam as Regras para Análise de Sementes (BRASIL, 1992).

A qualidade fisiológica das sementes foi avaliada através do teste de germinação, conforme metodologia descrita por BISOGNIN et al. (1991), e pêlos testes de vigor: primeira contagem de germinação $\left(4^{\circ}\right.$ dia) e tempo médio de germinação (TMG), em quatro repetições de 50 sementes. Estes testes foram realizados em sementes recém extraídas, após a secagem ao ar e aos 60 dias de armazenamento. O TMG foi calculado através da fórmula proposta por HARRINGTON (1972), sendo:

$$
T M G=\left(N_{l} T_{1}+N_{2} T_{2}+\ldots+N_{n} T_{n}\right) /\left(N_{l}+N_{2}+\ldots+N_{n}\right)
$$

onde: $\mathrm{N}$ é o número de sementes germinadas e $\mathrm{T}$ é o tempo em dias.

O experimento fatorial ( 3 tamanhos $\times 3$ épocas de avaliação) foi analisado segundo o delineamento completamente casualizado, com 4 repetições, sendo as médias comparadas pelo teste de Tukey, em nível de 5\% de probabilidade. Os dados de número de sementes/fruto foram transformados para raiz quadrada de $X+0,5$ e os dados de primeira contagem e de germinação para arco seno.

Experimento II - Sementes extraídas de frutos médios, 60 dias após a colheita, foram submetidas a dois métodos de remoção da placenta: fermentação em água e imersão em solução contendo $\mathrm{HC} 1$ a $25^{\circ} \mathrm{C}$. Os tratamentos de fermentação em água foram: 24,48, 72,96 e 120h na proporção de 1:5 (semente + placenta:água). Os tratamentos a base de $\mathrm{HC} 1$ consistiram na imersão das sementes em solução com $\mathrm{HC} 1$ por 30 min e homogeneização a cada 10min, na proporção de 1:2,5 (semente + placenta:solução) nas concentrações de 10,20 e $30 \%$ de $\mathrm{HC} 1$. A testemunha consistiu na retirada manual da placenta aderida às sementes.
Após a aplicação dos tratamentos, as sementes foram secas ao ar e avaliadas quanto a umidade, germinação e vigor, conforme descrito no Experimento I.

Os dados obtidos foram analisados pelo teste de $\mathrm{F}$ e os contrastes pelo teste de Scheffé. Também foi realizada a análise de regressão polinomial para a avaliação dos efeitos dos tratamentos de fermentação em água. $\mathrm{O}$ experimento foi analisado segundo o delineamento completamente casualizado.

\section{RESULTADOS E DISCUSSÃO}

Experimento I - Pela análise da Tabela 1, verifica-se as relações existentes entre o tamanho de fruto e características avaliadas tanto no fruto quanto na semente. $\mathrm{O}$ tamanho do fruto de porongo pode ser caracterizado pelo seu comprimento ou pelo diâmetro. Os frutos grandes e médios não apresentaram diferença significativa quanto a espessura do casco. Considerando que frutos médios apresentam maior valor comercial e que boa espessura do casco confere maior qualidade à cuia (BISOGNIN, 1989), o fato de frutos médios e grandes não diferirem significativamente quanto a espessura de casco, sugere que devem ser buscadas alternativas para reduzir o tamanho do fruto. Tais alternativas podem ser genéticas ou relacionadas ao manejo da cultura. BISOGNIN et al. (1992) constataram que o aumento da densidade de semeadura diminui o volume do fruto.

As diferenças obtidas entre os tratamentos mostraram uma relação direta entre tamanho de fruto e algumas características das sementes, pois quanto maior o tamanho dos frutos maiores são o número e a largura das sementes, conforme já havia sido indicado por BLEASDALE (1977) ao revisar o crescimento de fruto em diversas espécies. Este autor justificou tal afirmação pelo fato das sementes serem ricas fontes de giberelinas, que produzem marcado aumento no tamanho dos frutos. Quanto à espessura das sementes, não houve diferença significativa entre frutos grandes e médios. Já o comprimento da semente não foi modificado pelo tamanho de fruto. O peso de 1000 sementes decresceu com a diminuição do tamanho do fruto, portanto, frutos pequenos continham sementes mais leves devido, principalmente, a sua menor largura e espessura.

Com base nestas informações é possível quantificar a necessidade de sementes para a implantação de uma lavoura de porongo. Considerando uma densidade média de 13.300 plantas/ha e uma germinação mínima de $80 \%$, seria necessária a extração de sementes de 73 frutos grandes, ou de 101 médios, ou 
Tabela 1 - Características de futuros e de sementes de porongo segundo os diferentes tamanhos. Santa Maria, RS, 1996.

\begin{tabular}{|c|c|c|c|c|c|c|c|c|}
\hline & \multicolumn{3}{|c|}{ Característica do Fruto } & \multicolumn{5}{|c|}{ Característica da Semente } \\
\hline & $\begin{array}{l}\text { Comprimento } \\
(\mathrm{cm})\end{array}$ & $\begin{array}{l}\text { Diâmetro } \\
(\mathrm{cm})\end{array}$ & $\begin{array}{l}\text { Espessura } \\
\quad(\mathrm{cm})\end{array}$ & Número & $\begin{array}{l}\text { Comprimento } \\
(\mathrm{cm})\end{array}$ & $\begin{array}{l}\text { Largura } \\
\qquad(\mathrm{cm})\end{array}$ & $\begin{array}{l}\text { Espessura } \\
\quad(\mathrm{cm})\end{array}$ & $\begin{array}{c}\text { Peso } 1000 \\
(\mathrm{~g})\end{array}$ \\
\hline Grande & $11,85 \mathrm{a}^{*}$ & $52,15 \mathrm{a}$ & $0,85 \mathrm{a}$ & $288,72 \mathrm{a}$ & $1,52 \mathrm{a}$ & $0,71 \mathrm{a}$ & $0,30 \mathrm{a}$ & 200,73 \\
\hline Médio & $10,05 \mathrm{~b}$ & $44,15 \mathrm{~b}$ & $0,77 \mathrm{a}$ & $164,49 \mathrm{~b}$ & $1,49 \mathrm{~b}$ & $0,67 \mathrm{~b}$ & $0,29 \mathrm{a}$ & 161,34 \\
\hline Pequeno & $7,60 \mathrm{c}$ & $32,47 \mathrm{c}$ & $0,62 \mathrm{~b}$ & $95,70 \mathrm{c}$ & $1,36 \mathrm{a}$ & $0,63 \mathrm{c}$ & $0,27 \mathrm{~b}$ & 140,29 \\
\hline Média & 9,83 & 42,92 & 0,75 & 162,97 & 1,46 & 0,67 & 0,29 & 167,45 \\
\hline C V \% & 6,96 & 5,74 & 12,09 & 13,33 & 10,62 & 4,53 & 6,26 & - \\
\hline
\end{tabular}

*Tamanho de fruto com médias não seguidas pela mesma letra diferem entre si pelo teste de Tukey, a 5\% de probabilidade.

de 174 pequenos, o que equivale a um total de 3,34 ; 2,68 e $2,33 \mathrm{~kg}$ de sementes, respectivamente.

A análise da variação acusou interação altamente significativa $(\mathrm{P}<0,01)$ para todas as variáveis analisadas (Tabela 2). A avaliação do vigor das sementes oriundas de diferentes tamanhos de frutos acusou diferenças significativas entre os mesmos, mostradas pelo teste de primeira contagem, após a secagem ao ar e 60 dias depois, tais diferenças também foram registradas pelo TMG, no momento da extração das sementes. $\mathrm{O}$ armazenamento mostrou-se benéfico na melhoria da qualidade fisiológica das sementes, pois após 60 dias de armazenamento das sementes, em condições de laboratório, as mesmas produziram um maior número de plântulas normais e reduziram o TMG. Estes resultados indicam a presença de dormência, que é eliminada durante o armazenamento, concordando com as indicações de CASALI $\boldsymbol{e} \boldsymbol{t} \boldsymbol{a l}$. (1982), que considera que as sementes de cucurbitáceas podem apresentar dormência de até 30 dias após a sua extração. As sementes, por ocasião da extração, apresentaram umidade de 56 a $60 \%$, que após 15 dias baixou para aproximadamente $10 \%$.

Os resultados deste experimento mostram que, em porongo, o tamanho do fruto define algumas características, tanto para o fruto quanto para as sementes. Dentre as características de semente de maior importância prática, o número médio de sementes/fruto e o peso de 1000 sementes apresentaram relação direta com o tamanho do fruto, ou seja, frutos grandes contêm sementes maiores e mais pesadas. Estes resultados concordam em parte com os obtidos por INCALCATERRA et al. (1994) em melão, os quais verificaram a existência de uma redução significativa no peso de fruto, número de sementes/fruto, peso de sementes e percentagem de germinação com o aumento do número de ordem do

Tabela 2 - Percentagem de plântulas normais e tempo médio de germinação (TMG) de lotes de sementes oriundas de diferentes tamanhos de frutos em três épocas de avaliação. Santa Maria, RS, 1996.

\begin{tabular}{|c|c|c|c|c|}
\hline & \multirow{2}{*}{$\begin{array}{c}\text { Umidade } \\
(\%)\end{array}$} & \multicolumn{2}{|c|}{ Plântulas Normais (\%) } & \multirow{2}{*}{$\begin{array}{l}\text { TMG } \\
\text { (dias) }\end{array}$} \\
\hline & & $1^{\mathrm{a} C o n t a g e m}$ & Germinação & \\
\hline & \multicolumn{4}{|c|}{ Na Extração das Sementes } \\
\hline Grande & 58,95 & $8,42 \mathrm{a} \quad \mathrm{C}^{*}$ & $23,52 \mathrm{a} \quad \mathrm{C}$ & $7,04 \mathrm{a} A$ \\
\hline Médio & 56,11 & $9,18 \mathrm{a} \quad \mathrm{C}$ & $23,44 a \quad C$ & $7,06 \mathrm{a} A$ \\
\hline \multirow[t]{2}{*}{ Pequeno } & 60,18 & $10,34 \mathrm{a} C$ & $19,64 \mathrm{a} \quad \mathrm{C}$ & $4,98 \mathrm{~b} \mathrm{~A}$ \\
\hline & \multicolumn{4}{|c|}{ Após Secagem ao Ar } \\
\hline Grande & 10,54 & $53,46 \mathrm{a} \mathrm{B}$ & $61,48 \mathrm{a} \quad \mathrm{B}$ & $3,58 \mathrm{a} B$ \\
\hline Médio & 10,40 & $53,46 \mathrm{a} \mathrm{B}$ & $58,98 \mathrm{ab} \mathrm{B}$ & $3,41 \mathrm{a} \mathrm{B}$ \\
\hline \multirow[t]{2}{*}{ Pequeno } & 9,71 & $42,22 \mathrm{~b}$ B & $46,86 \mathrm{~b} \quad \mathrm{~B}$ & $4,17 \mathrm{a} A B$ \\
\hline & \multicolumn{4}{|c|}{ Após 60 dias } \\
\hline Grande & 10,34 & $85,48 \mathrm{ab} \mathrm{A}$ & $93,50 \mathrm{a} \quad \mathrm{A}$ & $3,12 \mathrm{a} \mathrm{B}$ \\
\hline Médio & 12,19 & $95,50 \mathrm{a} \quad \mathrm{A}$ & $98,00 \mathrm{a} \quad \mathrm{A}$ & $2,44 \mathrm{a} \quad \mathrm{B}$ \\
\hline Pequeno & 11,65 & $83,00 \mathrm{~b} \quad \mathrm{~A}$ & $93,00 \mathrm{a} \quad \mathrm{A}$ & $3,10 \mathrm{a} \mathrm{B}$ \\
\hline Média & 26,67 & 49,01 & 57,60 & 4,32 \\
\hline $\mathrm{CV} \%$ & - & 6,59 & 7,37 & 16,24 \\
\hline
\end{tabular}

*Tratamentos com médias não seguidas pela mesma letra maiúscula entre épocas, dentro de tamanho de fruto e minúscula entre tamanho de fruto dentro de época diferem pelo teste de Tukey, a $5 \%$ de probabilidade. 
fruto na planta, ou seja, a posição do fruto na planta tem efeito marcante na qualidade das sementes.

Experimento II - Com relação ao método de extração das sementes (Tabela 3) constata-se que a imersão das sementes + placenta em solução contendo $\mathrm{HC} 1$ nas concentrações de 20 e $30 \%$ reduziu a percentagem de plântulas normais e aumentou o TMG. O teste de Schefeé mostrou diferenças altamente significativas $(\mathrm{P}<0,01)$ como efeito de tratamento para primeira contagem e TMG e não significativas apenas para a germinação no contraste $\mathrm{C}_{1}$ Quando são comparados os efeitos dos tratamentos de HC1 com a testemunha, observou-se que este método não é adaptado para a extração de sementes de porongo, por ter reduzido sifnificativamente a germinação e/ou o vigor. Estes resultados discordam daqueles obtidos por COUTO et al. (1969), que constataram que a extração química $(\mathrm{HCl}$ ou $\mathrm{NaOH})$ de sementes de pepino foi mais rápida do que com o uso da fermentação e as sementes apresentaram-se mais

claras. Esta discordância entre os resultados provalvelmente está associada as diferenças de natureza da placenta e do pericarpo existentes entre as duas \espécies. Por outro lado, a fermentação em água proporcionou aumentos significativos na germinação e no vigor das sementes de porongo, comparado tanto com a testemunha quanto com a imersão em $\mathrm{HC} 1$.

O tempo de fermentação em água demonstrado pelo ajustamento das curvas de regressão polinomial (Figura 1) que proporcionou os melhores resultados em termos de germinação e vigor nas sementes de porongo foi: $70,4 \mathrm{~h}$ pela primeira contagem, 70,9 h pela germinação e $88,4 \mathrm{~h}$ pelo $\mathrm{TMG}$, o que correspondeu a 64,8 e 67,9\% de plântulas normais e 2,3 dias respectivamente. O aumento da qualidade das sementes de porongo parece estar associado ao fato de que, durante o processo de lavagem, é feita a retirada do sobrenadante, o que elimina as sementes vazias e/ou com cotilédones mal formados.

A fermentação pode apresentar desvantagens durante o processo de extração das sementes de frutos carnosos (SILVA, 1983), o que não foi verificado neste trabalho, pois este método facilitou a limpeza, melhorou o aspecto das sementes de porongo e até aumentou a qualidade fisiológica das sementes, quando comparado com a extração manual, que é demorada e na prática é inviável. Entre os tratamentos de fermentação em água, aqueles que proporcionaram uma fácil limpeza das sementes através de uma leve fricção, retirada do sobrenadante e lavagem foram os de $48,72 \mathrm{e}$ 96h. Com apenas 24h de fermentação, a placenta se apresentava ainda firme e aderida às sementes, indicando a necessidade de um maior período de fermentação. No tratamento de $120 \mathrm{~h}$, a placenta encontrava-se em estado de decomposição, o fermentado com cheiro desagradável e ocorreu o início da germinação das sementes, sendo indicativos do período ter sido muito

Estimativas dos Contrastes

Contrastes
Test.x Trat $\left(\mathrm{C}_{1}\right)$

Tesx Água $\left(\mathrm{C}_{2}\right)$

Tes. $\mathrm{x} \mathrm{HCl}\left(\mathrm{C}_{3}\right)$

ÁguaxHCl $\left(\mathrm{C}_{4}\right)$

$\begin{array}{ccc}-97,40 & -13,02 \mathrm{~ns} & -6,11^{*} \\ 192,72 & -95,38^{* *} & 3,98 \\ 95,32 & 82,36^{* *} & -10,02 \\ 1054,7 & 697,94 & -61,83\end{array}$

* significado pelo teste de Scheffé, a $1 \%$ de probabilidade ns $=$ não significado longo.

A curva de durante os tratamentos de fermentação em água, está representado na Figura 2. Observa-se uma rápida absorçã 
de água na primeira hora, chegando a $34,0 \%$ de umidade, mantendo-se assim até a hora seguinte. Posteriormente, a velocidade de absorção diminuiu, determinando pequenos acréscimos na umidade das sementes até atingir $44,0 \%$ após 12 horas de embebição. A umidade se manteve praticamente constante entre 12 e $120 \mathrm{~h}$ de fermentação. Este comportamento ajusta-se ao descrito por BEWLEY \& BLACK (1985), onde a embebição ocorre de acordo com um padrão trifásico, com uma primeira fase de hidratação rápida, seguida de outra mais longa de aparente repouso, em que a hidratação é lenta, voltando a hidratação rápida na fase três.

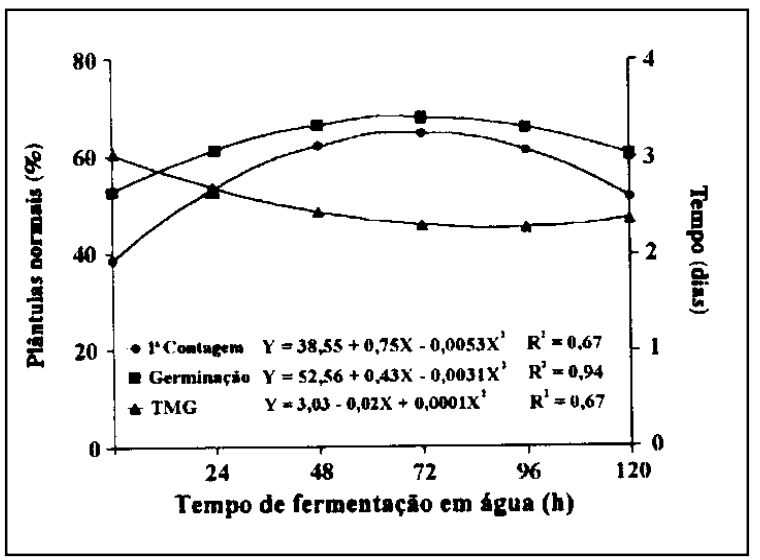

Figura 1 - Desempenho de sementes de porondo submetido a fermentação em água a $25^{\circ} \mathrm{C}$ por diferentes tempo. Santa Maria, RS, 1996.

No teste de germinação acompanhou-se a absorção de umidade pelas sementes, verificando-se que a germinação visível, ou seja, a profusão da raiz primária, somente ocorreu após aquelas terem atingido a umidade média de 43,4\%. Quando determinou-se a umidade das diversas partes da semente, constatou-se $49,5 \%$ de umidade na casca e $30,3 \%$ no embrião.

Durante o processo de fermentação, a germinação das sementes não ocorreu mesmo após $44,0 \%$ de umidade, provavelmente, devido a presença de inibidores de germinação presentes na placenta e/ou a deficiência de oxigénio no fermentado.

\section{CONCLUSÕES}

1. Quanto maior o porongo maior é o número e peso de suas sementes, sem diferença na capacidade de produzir plântulas normais.

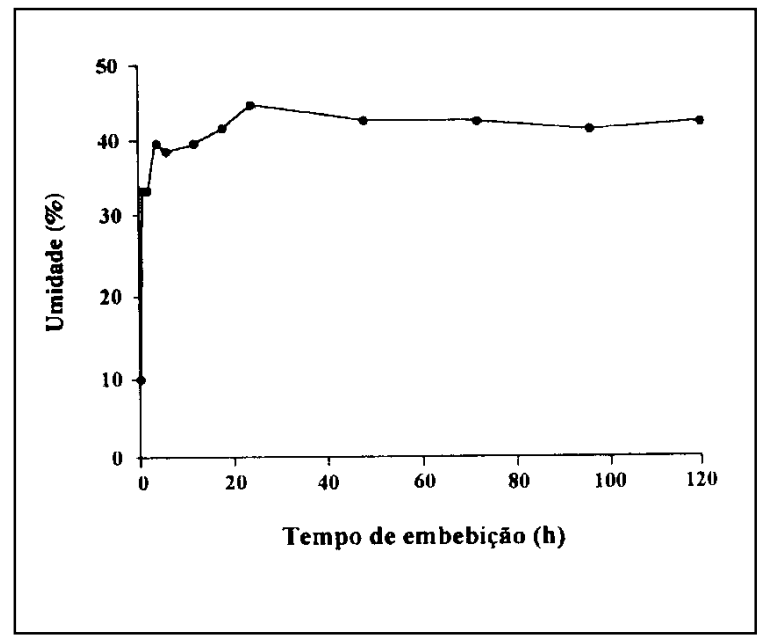

Figura 2 - Curva de embebição de sementes de porongo em água a $25^{\circ} \mathrm{C}$. Santa Maria, RS, 1996.

2. A limpeza de sementes de porongo através da imersão em solução contendo HC1 reduz o vigor.

3. O melhor tempo de fermentação para as sementes de porongo em água à $25^{\circ} \mathrm{C}$ é de $72 \mathrm{~h}$, no entanto o período de 48 a $96 \mathrm{~h}$ melhora a qualidade fisiológica, além de facilitar a limpeza.

4. As sementes de porongo, em condições favoráveis de temperatura, umidade e aeração, germinam após terem atingido 43,4\% de umidade.

\section{REFERÊNCIAS BIBLIOGRÁFICAS}

ALVARENGA, E. M., SILVA, R.F., ARAÚJO, E.F. $\boldsymbol{e} t \boldsymbol{a l}$ Influência da idade e armazenamento pós-colheita dos frutos na qualidade de sementes de melancia. Horticultura Brasileira, Brasília, v. 2, n. 2, p. 5-8, 1984.

ARAÚJO, E. F., MANTOVANI, E. C., SILVA, R. F. da. Influência da idade e armazenamento dos frutos na qualidade de sementes de abóbora. Revista Brasileira de Sementes, Brasília, v. 4, n. 4,p.77-87, 1982.

BEWLEY, J.D., BLACK, M. Seeds: Physiology ofdevelopment and germination. New York: Plenum, 1985. 367 p.

BISOGNIN, D. A., MARCHESAN, E. Avaliação de algumas populações de porongo - Lagenaria siceraria (Mol.) Standi, cultivadas na região de Santa Maria, RS. Revista do Centro de Ciências Rurais, Santa Maria, v. 18, n. 3-4, p. 201-207, 1988.

BISOGNIN, D. A. Avaliação e classificação da cuia para indústria. In: ENCONTRO DE BIOLOGIA, 1, 1989. UFPel, Pelotas. Anais... Pelotas, Instituto de Biologia, 1989, 103 p. p.7.

BISOGNIN, D. A., 1RIGON, D. L., MARTINAZZO, A. A. Teste de germinação em porongo - Lagenaria siceraria (Mol.) Standi. Ciência Rural, Santa Maria, v. 21, n. 2, p. 159-167, 1991.

Ciência Rural, v. 27, n. 1, 1997. 
BISOGNIN, D. A., MARCHESAN, E., AUDE, M. I. da S. Densidade de semeadura e produtividade do porongo. Ciência Rural, Santa Maria, v. 22, n. 1, p. 15-19, 1992.

BISOGNIN, D. A., AMARANTE, C. V. T. do, NICHIMORI, K H. Análise do crescimento inicial de plantas de porongo Lagenaria siceraria. Horticultura Brasileira, Brasília, v. 13, n.2,p.163-166, 1995

BLEASDALE, J. K. A. Fisiologia Vegetal. Trad. WEISHAUPL, L. \& LAMBERTI, A. São Paulo: EPU-EDUSP, 1977. 169 p.

BRASIL. Ministério da Agricultura e Reforma Agrária, Departamento Nacional de Produção Vegetal. Divisão de Sementes e Mudas. Regras para Análise de Sementes. Brasília: Ministério da Agricultura, 1992. 365 p.

CASAli, V. W. D., SATURNiNO, H. M., PEDROSA, J. F. Botânica e origem das cucurbitáceas. In: EPAMIG. As cucurbitáceas. Informe Agropecuàrio, Belo Horizonte, v. S, n. 85, p.22-23, 1982.
COUTO, F. A. A., SIlvA, R. F., SIlVA, J. F. Ensaio sobre métodos de extração de sementes de pepino (Cucumis sativus L.). Revista Ceres, Viçosa, v. 16, n. 17, p. 57-62, 1969.

HARRINGTON, J. F. Seed Storage Longevity. In: KOZLOSKI, T. T. Seed biology, New York: Academic, 1972. v. 3. p. 145245.

INCALCATERRA, G., CARUZO, P., QUAGLIOTTI, L. et al Seed quality ofwinter melon (Cucumis melo L. var. Inodorus Nand) as influenced by the position of fruits on the mother plant. Acta Horticulturae, n.362, p. 113-116, 1994

NASCIMENTO, W. N., PESSOA, H. B. S. V., SILVA, J. B. C. Remoção da mucilagem e seus efeitos na qualidade das sementes de pepino e tomate. Horticultura Brasileira, Brasília, v. 12, n.2,p.169-172, 1994.

SILVA, R. F. da. Extração de Sementes de Frutos Carnosos. In CARVALHO, N. M., NAKAGAWA, J. (ed.). Sementes: Ciência, Tecnologia e Produção 2. ed. rev. Campinas: Fundação Cargill, 1983. p. 379-404. 